\title{
Identificação e monitorização de cochonilhas-algodão (Hemiptera, Pseudococcidae) associadas ao cafeeiro em estufa
}

\section{Identification and monitoring of mealybugs (Hemiptera, Pseudococcidae) associated with coffee under greenhouse conditions}

\author{
Adilma Andrade ${ }^{1}$ Elsa Borges da Silva1,2,*, Ana Paula Pereira³ e José Carlos Franco ${ }^{1,2}$ \\ ${ }^{1}$ Departamento de Ciências e Engenharia de Biossistemas, Instituto Superior de Agronomia, Universidade de Lisboa, 1349-017 Lisboa, Portugal \\ ${ }^{2}$ Centro de Estudos Florestais, Instituto Superior de Agronomia, Universidade de Lisboa, 1349-017 Lisboa, Portugal \\ ${ }^{3}$ CIFC - Centro de Investigação das Ferrugens do Cafeeiro, Instituto Superior de Agronomia, Universidade de Lisboa, Oeiras, Portugal \\ ( ${ }^{*}$-mail: elsasilva@isa.ulisboa.pt) \\ http://dx.doi.org/10.19084/RCA16144
}

Recebido/received: 2016.11.02

Aceite/accepted: 2017.01.27

\section{R E S U M O}

O presente trabalho teve como principais objetivos: a) identificar as espécies de cochonilhas-algodão presentes em genótipos de cafeeiro utilizados como variedades comerciais em diferentes países cafeicultores, Catimor e Sarchimor (derivados do Híbrido de Timor), mantidos em estufa, no Centro de Investigação das Ferrugens do Cafeeiro, Oeiras; b) estudar a dinâmica de infestação de Planococcus citri (Risso) através de observação visual e da monitorização do voo dos machos com armadilhas sexuais, em cafeeiros das variedades Catimor e Sarchimor, em estufa; e c) comparar a taxa de colonização e o desenvolvimento de $P$. citri em dois genótipos de cafeeiro, um suscetível à ferrugem-alaranjada (Caturra vermelho CIFC 19/1) e outro resistente (Catimor CIFC 45).

Foram identificadas duas espécies de cochonilhas-algodão, Phenacoccus madeirensis Green e P. citri. A variedade Catimor foi a mais suscetível a $P$. citri. Não se detetaram diferenças significativas na duração do desenvolvimento de $P$. citri nas duas variedades estudadas, no entanto a taxa de colonização foi sempre superior na variedade Caturra CIFC 19/1.

Palavras-chave: Coffea arabica, Planococcus citri, Phenacoccus madeirensis, Pseudococcidae, Portugal.

\section{A B S T R A C T}

The aims of the present work were: a) to identify the mealybug species associated with commercial varieties of coffee, Catimor and Sachimor, in the greenhouses of Centro de Investigação das Ferrugens do Cafeeiro, Oeiras, Portugal; b) to study the dynamics of infestation of Planococcus citri (Risso), by visual observation and pheromone male trapping in the varieties Catimor and Sachimor, under greenhouse conditions; and c) to compare the colonization rate and development of $P$. citri in two coffee genotypes, one susceptible to coffee leaf rust (Caturra vermelho CIFC 19/1) and one resistant (Catimor CIFC 45).

Two species of mealybugs were identified, Phenacoccus madeirensis Green and P. citri. The variety Catimor was the most susceptible to $P$. citri. No significant differences were observed in the development time of $P$. citri among the studied varieties. Nevertheless, the rate of colonization by mealybugs was higher in Caturra CIFC 19/1.

Keywords: Coffea arabica, Planococcus citri, Phenacoccus madeirensis, Pseudococcidae, Portugal. 


\section{INTRODUÇÃO}

O café constitui um dos produtos agrícolas mais valiosos, para muitos países tropicais e subtropicais, que dependem da cafeicultura para obtenção de divisas e equilíbrio da sua balança comercial. De acordo com a Organização Internacional do Café (ICO, 2016), a produção mundial de café verde totalizava cerca de 8,9 milhões de toneladas, em 2015/16. No entanto, o cafeeiro (Coffea spp.) é uma cultura muito suscetível a pragas e doenças. São conhecidas mais de 850 espécies de insetos associadas ao cafeeiro, mas apenas algumas têm importância económica (Barrera, 2008). As cochonilhas-algodão são um dos grupos de pragas do cafeeiro, estando referenciadas mais de 50 espécies, na cultura (Ben-Dov, 2014). Os prejuízos provocados por algumas espécies de cochonilhas-algodão podem atingir a totalidade da produção, em casos de infestações mais severas (Santa-Cecília et al., 2002). Estas cochonilhas constituem fator limitante para cafeeiros jovens e adultos e a sua disseminação é muito rápida, dado que tanto as ninfas, como as fêmeas adultas são móveis, podendo as ninfas jovens ser transportadas pelo vento ou dispersar, por locomoção, para plantas vizinhas. Excretam melada, o que facilita o aparecimento de fungos saprófitas (fumagina), além de atrair formigas, que podem proteger as cochonilhas dos seus inimigos naturais e auxiliar na sua dispersão (Fornazier et al., 2000). O seu comportamento críptico e o facto de apresentarem o corpo revestido por secreções cerosas limitam a eficácia da luta química. Entre as espécies mais importantes, destacam-se Planococcus citri (Risso), Planococus lilacinus (Cockerell), Pseudococcus longispinus (Targioni-Tozzetti) e Planococcus kraunhiae (Kuwana) (Santa-Cecília et al., 2005; Kumar et al., 2008; Rahiman et al., 2010; Thuy et al., 2011).

O presente trabalho foi realizado no Centro de Investigação das Ferrugens do Cafeeiro, (CIFC), em Oeiras. O CIFC tem como principal objetivo contribuir para o desenvolvimento de variedades de cafeeiro com resistência à ferrugem-alaranjada, Hemileia vastatrix Berk. \& Broome (Pereira \& Guimarães, 2010). Para o efeito, possui uma área de cerca de 0,5 ha de estufas aquecidas, nas quais são mantidas as suas coleções de variedades de cafeeiro. Um dos principais problemas fitossanitários das plantas mantidas nas estufas do CIFC, que limita grandemente a obtenção do material vegetal necessário às inoculações dos fungos aí estudados, são as cochonilhas-algodão. Este trabalho teve como principais objetivos: a) identificar as espécies de cochonilhas-algodão presentes em genótipos de cafeeiro utilizados como variedades comerciais em diferentes países cafeicultores, como Catimor e Sarchimor (derivados do Híbrido de Timor), mantidos em estufa, no CIFC; b) estudar a dinâmica de infestação de $P$. citri, através de observação visual e da monitorização do voo dos machos com armadilhas sexuais, em cafeeiros das variedades Catimor e Sarchimor, em estufa; c) comparar a taxa de colonização e o desenvolvimento de P. citri em dois genótipos de cafeeiro, um resistente à ferrugem-alaranjada (Catimor CIFC 45) e outro suscetível (Caturra CIFC 19/1).

\section{MATERIAL E MÉTODOS}

\section{Identificação das espécies}

Entre outubro de 2013 e abril de 2014, foram colhidas amostras de cochonilhas-algodão, em plantas infestadas, de diferentes variedades de cafeeiro, existentes nas estufas do CIFC (estufim 2 , tanque, estufas das culturas, cacaus). As cochonilhas que se encontravam no estado de fêmea adulta foram colocadas em tubos de Eppendorf em solução de conservação (1 parte de ácido acético e 3 partes de etanol a $96 \% \mathrm{v} / \mathrm{v})$, para posterior identificação. Os restantes indivíduos foram criados em brolhos de batata $\left(19^{\circ} \mathrm{C}-25^{\circ} \mathrm{C}\right)$, até atingirem o estado adulto, tendo sido depois mantidos em solução de conservação.

Os exemplares colhidos de cochonilhas-algodão foram posteriormente preparados em lâmina-lamela, segundo o método descrito por Williams \& Watson (1988), para proceder à sua identificação taxinómica, que foi feita com base em Williams \& Granara de Willink (1992) e, posteriormente, confirmada pelos Professores Agatino Russo e Gaetana Mazzeo (Universidade de Catania, Itália).

Foram, igualmente, obtidos parasitóides emergidos das cochonilhas amostradas e mantidas em laboratório, bem como amostras de formigas detetadas nas plantas infestadas, que foram conservados em etanol a 70\%, em tubos de Eppendorf, 
para posterior identificação. A identificação dos parasitóides foi feita pelos autores, com base em Triapitsyn et al. (2007), e a das formigas foi efetuada pela Mestre Vera Zina (ISA, Universidade de Lisboa), com base em Collingwood \& Prince (1998). Em ambos os casos, os exemplares foram estudados com auxílio de microscópio estereoscópico (50X; Nikon SMZ-2B).

\section{Monitorização da população de cochonilhas- -algodão em cafeeiro}

\section{Observação visual}

A monitorização do nível de infestação dos cafeeiros com cochonilhas-algodão decorreu de janeiro a junho de 2014, no Estufim 2 do $\operatorname{CIFC~}\left(377,1 \mathrm{~m}^{2}\right)$. Consistiu na observação visual de 112 plantas de C. arabica, semeadas em vasos individuais $(50 \mathrm{~cm}$ de diâmetro x $51 \mathrm{~cm}$ de altura), distribuídas em três blocos: 43 plantas de Sarchimor e cinco de Cavimor ( $\mathrm{n}=48)$, no primeiro bloco; 45 plantas de Catimor, uma de Sarchimor, uma de Cavimor e uma de Villa Sarchi $(\mathrm{n}=48)$, no segundo bloco; e 14 plantas de Catuaí, uma de Villa Sarchi e uma de Catimor ( $\mathrm{n}=16)$, no terceiro bloco. As variedades Caturra e Villa Sarchi de C. arabica são mutações para porte baixo de Bourbon, sendo que a primeira foi encontrada perto da cidade de Caturra, no Brasil, nos anos 1930 e a segunda, na Costa Rica. A variedade Catuaí de C. arabica é um híbrido de Caturra e Mundo Novo, criado no Brasil, nos finais dos anos 1940. Catimor é um híbrido obtido a partir do cruzamento artificial do cafeeiro Caturra Vermelho CIFC19/1 com Híbrido de Timor, HDT CIFC 832/1. O cruzamento entre Catuaí e Catimor deu origem à variedade Cavimor. A variedade Sarchimor é um híbrido entre Villa Sarchi e HDT CIFC 832/2.

O nível de infestação foi determinado, quinzenalmente, para cada planta, com base na seguinte escala: 0 - ausência de cochonilhas; 1 - infestação fraca (fêmeas isoladas, colónias ou ninfas isoladas); 2 - infestação moderada (presença de posturas, 1 e 2 instares em alguns ramos); 3 - infestação forte (presença de posturas e todos os estádios de desenvolvimento nos órgãos de todos ou quase todos os ramos).
Foi, ainda, avaliado o vigor das plantas, para posteriormente se analisar a possível relação entre vigor e nível de infestação, tendo-se considerado três classes: a) vigor elevado (todos os ramos com folhas); b) vigor médio (alguns ramos sem folhas); c) vigor fraco (folhas apenas nos ramos superiores da copa).

\section{Curva de voo dos machos}

A monitorização do voo dos machos de $P$. citri foi efetuada, entre 21 de janeiro e 18 de junho de 2014, através de armadilhas adesivas brancas $(15 \mathrm{~cm} x$ $15 \mathrm{~cm}$ ), ativadas com difusores de feromona sexual de síntese, comercializados pela empresa Biosani. Foram instaladas duas armadilhas, a $1 \mathrm{~m}-1,5 \mathrm{~m}$ de altura, no quadrante sudoeste, num ramo do interior da copa de duas plantas escolhidas ao acaso, distando $17 \mathrm{~m}$ entre si, para minimizar interferências. Os difusores foram substituídos de 4 em 4 semanas. As capturas dos machos foram contabilizadas semanalmente, por observação ao microscópio estereoscópico (20X; Nikon SMZ-2B).

As temperaturas médias diárias foram estimadas a partir das temperaturas máximas e mínimas registadas em termómetro (TFA, Germany) instalado na estufa.

\section{Comparação do desenvolvimento de Planococcus citri em duas variedades de cafeeiro, com diferente suscetibilidade à ferrugem-alaranjada}

Comparou-se o desenvolvimento de P. citri em duas variedades de C. arabica, com diferente suscetibilidade à ferrugem-alaranjada: Caturra vermelho - CIFC 19/1 (suscetível) e Catimor CIFC 45 (resistente). As plantas foram obtidas de semente e cresceram em vasos contendo uma mistura de terra preta, terra vegetal, turfa e areia na proporção 1:3:1:2 (v/v/v/v). A sementeira foi realizada em julho de 2013, tendo sido necessário aguardar cerca de três meses para o aparecimento do par de folhas cotiledonares. Doze plantas de cada variedade foram então transferidas para vasos individuais (16 cm de diâmetro x $15 \mathrm{~cm}$ de altura), com a mesma mistura referida anteriormente, mas na proporção de 1:3:1:1 (v/v/v/v).

A infestação das plantas com $P$. citri foi feita ao aparecimento dos dois primeiros pares de folhas. Cada planta foi infestada com fêmeas fecundadas, 
obtidas a partir de colónias criadas em brolhos de batata (Solanum tuberosum L.), em condições controladas $\left(24^{\circ} \mathrm{C} \pm 0,5^{\circ} \mathrm{C}, 60 \% \pm 10 \% \mathrm{HR}\right.$, fotoperíodo 00L:24E), provenientes de fêmeas em postura colhidas em laranjeira-doce, Citrus sinensis (L.) Osbeck, na região de Silves. As fêmeas foram desalojadas dos brolhos de batata, com auxílio de uma agulha entomológica, de modo a evitar que partissem os estiletes da armadura bucal, no processo de transferência para cafeeiro. A infestação foi feita numa sala própria para inoculações, tendo depois as plantas sido transferidas para uma estufa isolada, de modo a evitar contaminações.

As observações foram realizadas semanalmente, envolvendo a inspeção de cada planta, com o auxílio de uma lupa de bolso, para registar a evolução dos vários estados de desenvolvimento da cochonilha. Nas plantas com elevado número de ninfas do segundo instar, procedeu-se à eliminação de exemplares para evitar problemas de competição intraespecífica excessiva.

No final da primeira geração, mediu-se o tamanho das plantas e contabilizou-se o número de folhas por planta, com o objetivo de avaliar o vigor de cada uma das variedades. No final do ensaio, averiguou-se também a presença de cochonilhas nas raízes.

\section{Análise estatística}

Para a análise estatística, utilizou-se o teste-t de Student para duas amostras independentes, na comparação das médias do nível de infestação nas variedades Sarchimor e Catimor. A variedade Catuaí não foi considerada nesta análise, uma vez que a dimensão da amostra foi relativamente pequena (16 plantas), quando comparada com as outras duas (48 plantas). O mesmo teste foi utilizado para comparar o número médio de fêmeas de $P$. citri que atingiram o estado adulto nas duas variedades estudadas de cafeeiro.

$\mathrm{Na}$ análise, por uma questão de simplificação de linguagem, referem-se apenas as três variedades mais representativas, em cada um dos blocos estudados. Assim, o primeiro bloco foi designado de Sarchimor $(n=48)$, o segundo de Catimor $(n=48)$ e o terceiro de Catuaí ( $\mathrm{n}=16)$.
Os dados são apresentados sob a forma de média \pm erro padrão. Todas as análises foram realizadas através do programa IBM SPSS statistics 22.0 para Windows (Inc. Chicago, EUA).

\section{RESULTADOS}

\section{Identificação das espécies}

Os exemplares de cochonilhas-algodão colhidos nas estufas do CIFC foram identificados como pertencendo às espécies $P$. citri e Phenacoccus madeirensis Green, sendo aparentemente a primeira espécie a mais frequente, dominante e também a única que foi detetada no Estufim 2, onde decorreu o estudo da dinâmica de infestação.

Foram recolhidas três espécies de parasitóides do género Anagyrus Howard (Hymenoptera: Encyrtidae), nomeadamente $A$. sp. próx. pseudococci (Girault) e mais duas espécies não identificadas, associadas respetivamente a $P$. citri e $P$. madeirensis.

Identificaram-se três espécies de formigas em associação com cochonilhas, nomeadamente Lasius grandis (Forel) (em folhas de cafeeiro), Tapinoma simrothi (Krausse) (no tronco) e Crematogaster auberti (Emery).

\section{Monitorização da população de cochonilhas- -algodão em cafeeiros}

\section{Observação visual}

No início da monitorização, a variedade Sarchimor foi a que apresentou maior percentagem de plantas infestadas com $P$. citri, assim como maior índice médio de infestação (Figura 1B). Entre meados de janeiro e início de abril, a percentagem de plantas infestadas manteve-se relativamente estável nas variedades Catimor e Sarchimor, tendo-se a partir daí registado incremento deste parâmetro na variedade Catimor, que ultrapassou a variedade Sachimor antes do tratamento inseticida com clorpirifos, realizado a 16 de maio. A percentagem de plantas infestadas da variedade Catuaí manteve-se constante entre meados de janeiro e meados de maio (Figura 1B). Todas as variedades estudadas registaram diminuição acentuada da percentagem 
de plantas infestadas, após o tratamento inseticida, tendo este parâmetro atingido valores próximos de zero, nas variedades Sarchimor e Catuaí, no final de maio. Já no caso da variedade Catimor, a percentagem de plantas infestadas registou um mínimo de cerca de $20 \%$. Muito embora as observações tenham terminado em junho, os dados sugerem a existência de ressurgimento da infestação cerca de três semanas após o tratamento inseticida.

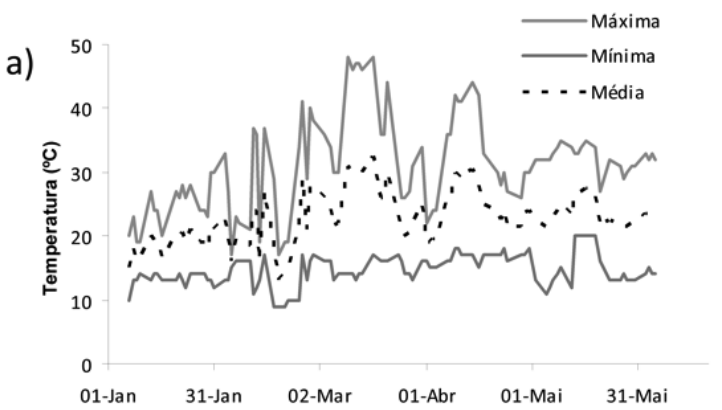

b) $100-$ Sarchimor $(n=48)-$ Catimor $(n=48)-$ Catuai $(n=16)$

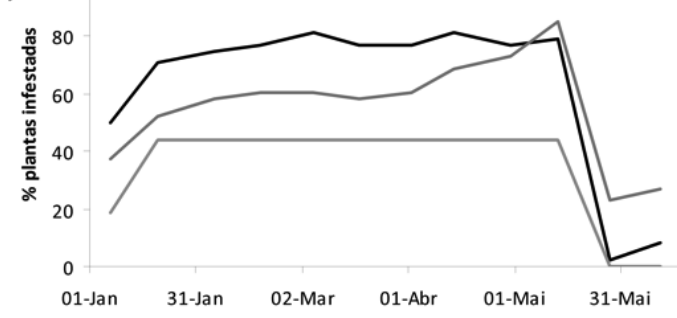

c)

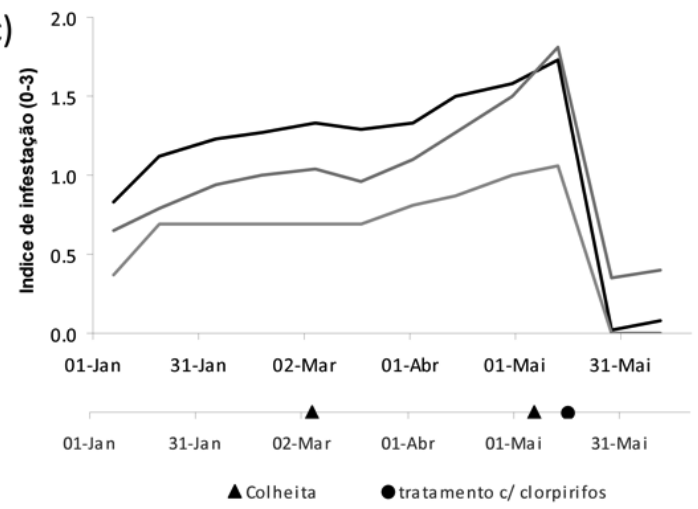

Figura 1 - Evolução da temperatura (A) e do nível de infestação por cochonilhas-algodão, medido em percentagem de plantas infestadas (B) e índice médio de infestação (C), nas três variedades de cafeeiro (Sarchimor, Catimor e Catuaí), existentes no estufim 2 do CIFC (Oeiras), e operações culturais $(D)$ realizadas.
O índice de infestação cresceu a ritmo idêntico nas variedades Sarchimor e Catimor, até meados de março (Figura 1C). A partir dessa data, o mesmo parâmetro cresceu de forma mais acentuada na variedade Catimor, que ultrapassou a variedade Sarchimor imediatamente antes da realização do tratamento inseticida. No caso da variedade Catuaí, o índice de infestação manteve-se constante entre meados de janeiro e meados de março e registou um aumento linear a partir daí. O comportamento deste parâmetro, após o tratamento inseticida, foi idêntico ao já referido para a percentagem de plantas infestadas.

A temperatura média no interior da estufa variou entre $13^{\circ} \mathrm{C}$ e $30^{\circ} \mathrm{C}$, durante o período experimental, tendo apresentado evolução crescente entre janeiro e meados de março e estabilizado a partir daí, embora com oscilações (Figura 1A).

Verificou-se existir forte relação linear entre a percentagem de plantas infestadas por cochonilhas-algodão e o índice médio de infestação para as variedades Sarchimor e Catimor (Figura 2). Não se observou a existência de relação significativa no caso da variedade Catuaí.

A análise da evolução da fenologia do cafeeiro (Figura 3) permitiu verificar que o maior número de plantas com frutos verdes foi registado em janeiro, para a variedade Sarchimor, e em fevereiro-março, para as variedades Catuaí e Catimor. Os frutos maduros foram mais frequentes em maio, na variedade Catuaí, em abril, na variedade Catimor, e em fevereiro-março, na variedade Sarchimor. Ocorreram dois períodos de diminuição rápida do número de plantas com frutos maduros. O primeiro ocorreu em março, nas três variedades, e o segundo em maio, apenas para a variedade Catimor e Sarchimor. Nota-se, no entanto, que esta diminuição foi sempre mais acentuada para a variedade Sarchimor. Embora, inicialmente, o maior número de plantas com flores tenha sido observado na variedade Sarchimor, a partir de abril, altura em que se verificou o maior número de flores na estufa, a variedade Catimor foi a que apresentou maior número de plantas em floração. 
a)

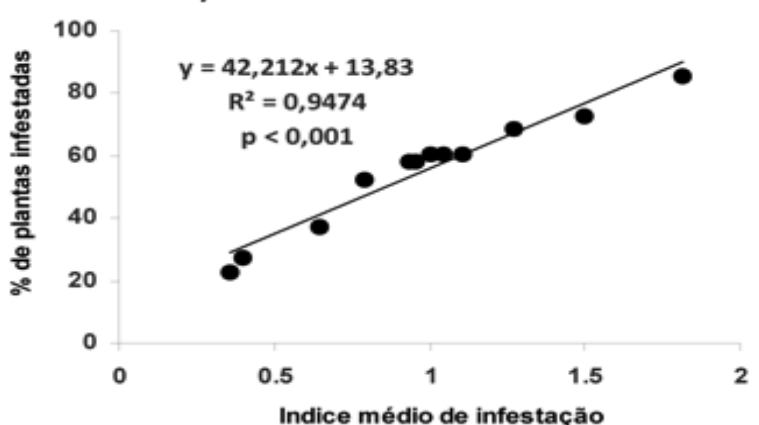

b)

Sarchimor

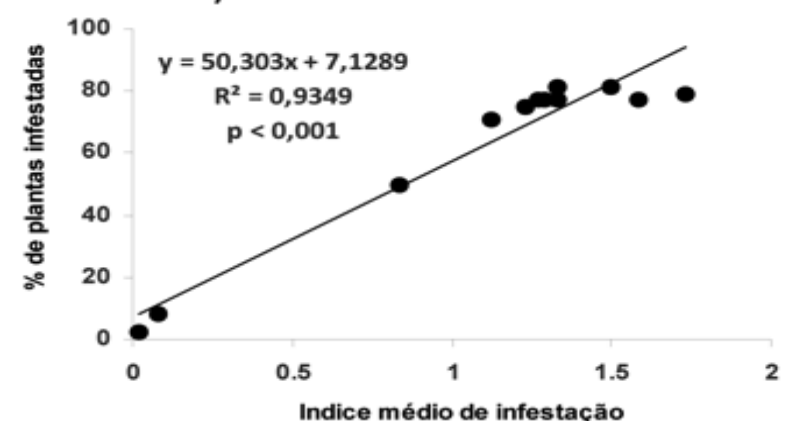

c) Catuai

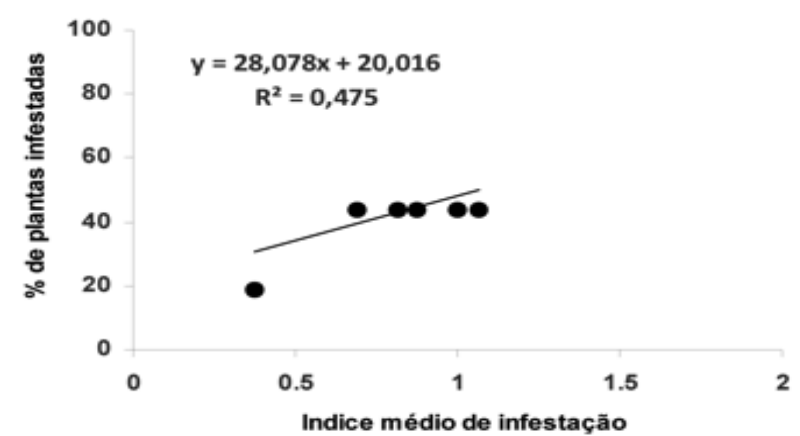

Figura 2 - Relação entre a percentagem de plantas infestadas com cochonilhas-algodão (Hemiptera, Pseudococcidae) e 0 índice médio de infestação para as variedades de cafeeiro estudadas no estufim 2 do CIFC (Oeiras): A- Catimor; B- Sarchimor; C- Catuaí.
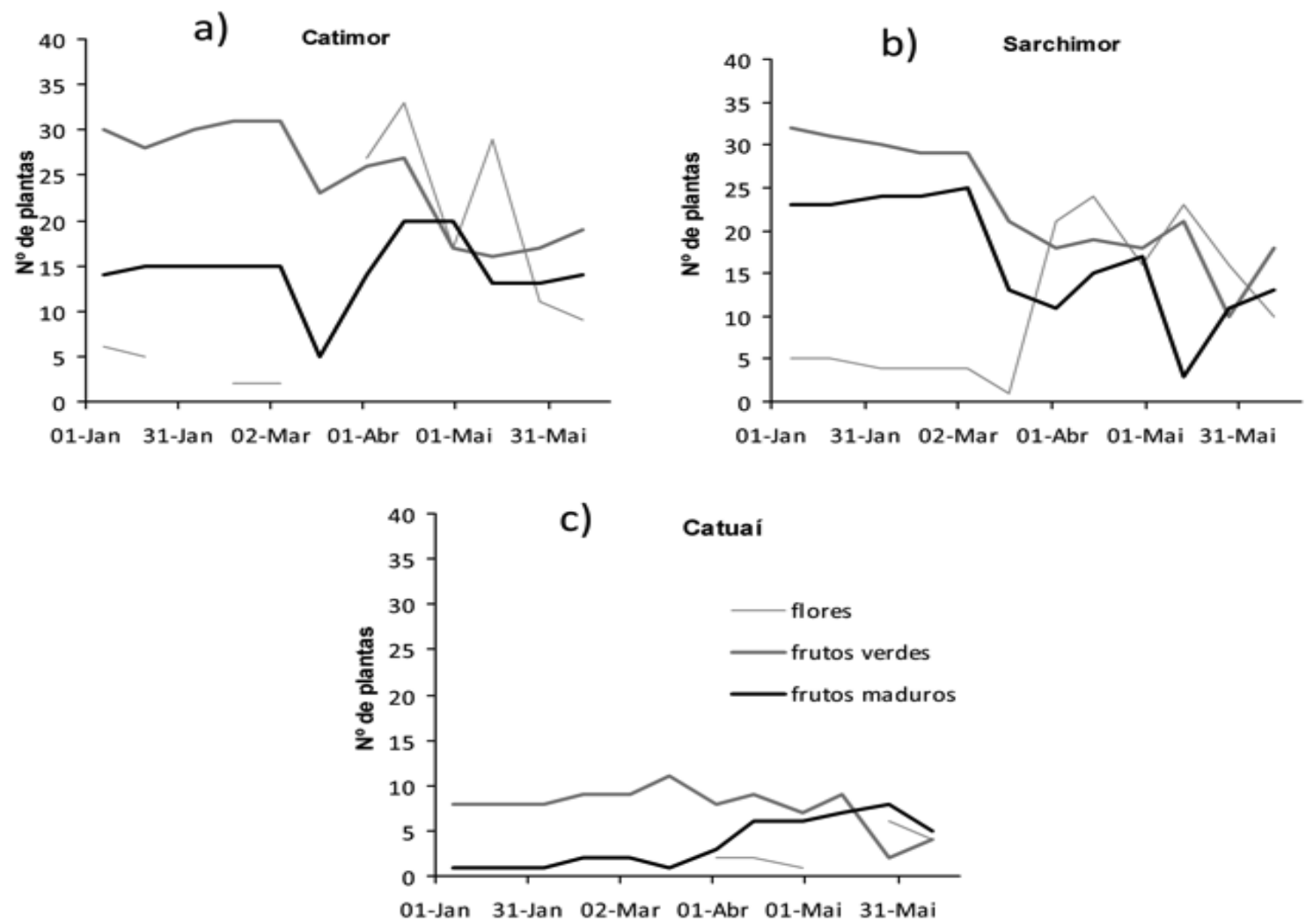

Figura 3 - Evolução dos estados fenológicos dominantes das variedades de cafeeiro estudadas no Estufim 2 do CIFC (Oeiras): A- Catimor; B- Sarchimor; C- Catuaí. 


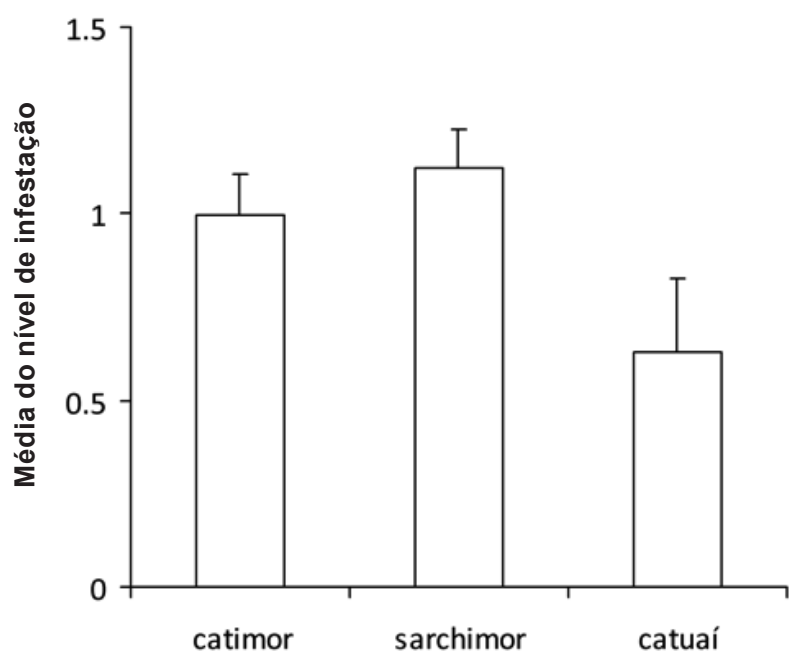

Figura 4 - Índice médio de infestação por cochonilhasalgodão nas três variedades de cafeeiro estudadas (Catimor, Sarchimor, Catuaí).
Não se registaram diferenças significativas $\left(\mathrm{t}_{94}=-\right.$ $-0,838, p=0,404)$ no nível médio de infestação entre as variedades Catimor e Sarchimor (Figura 4). $\mathrm{O}$ vigor vegetativo das plantas diferiu entre variedades, tendo a percentagem de plantas com elevado vigor sido máxima na variedade Sarchimor e mínima na variedade Catuaí (Figura 5).

O índice médio de infestação parece estar diretamente relacionado com a percentagem de plantas com vigor elevado e inversamente relacionado com percentagem de plantas com vigor fraco (Figura 5).

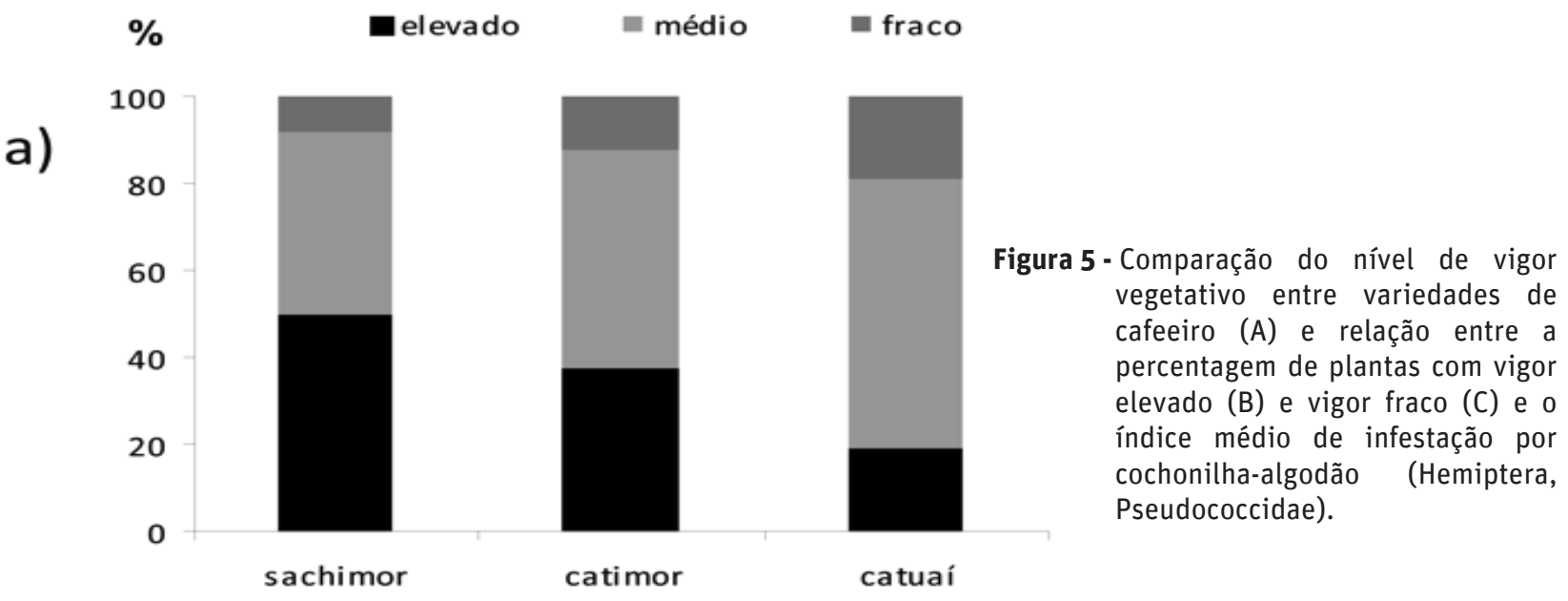

Variedades
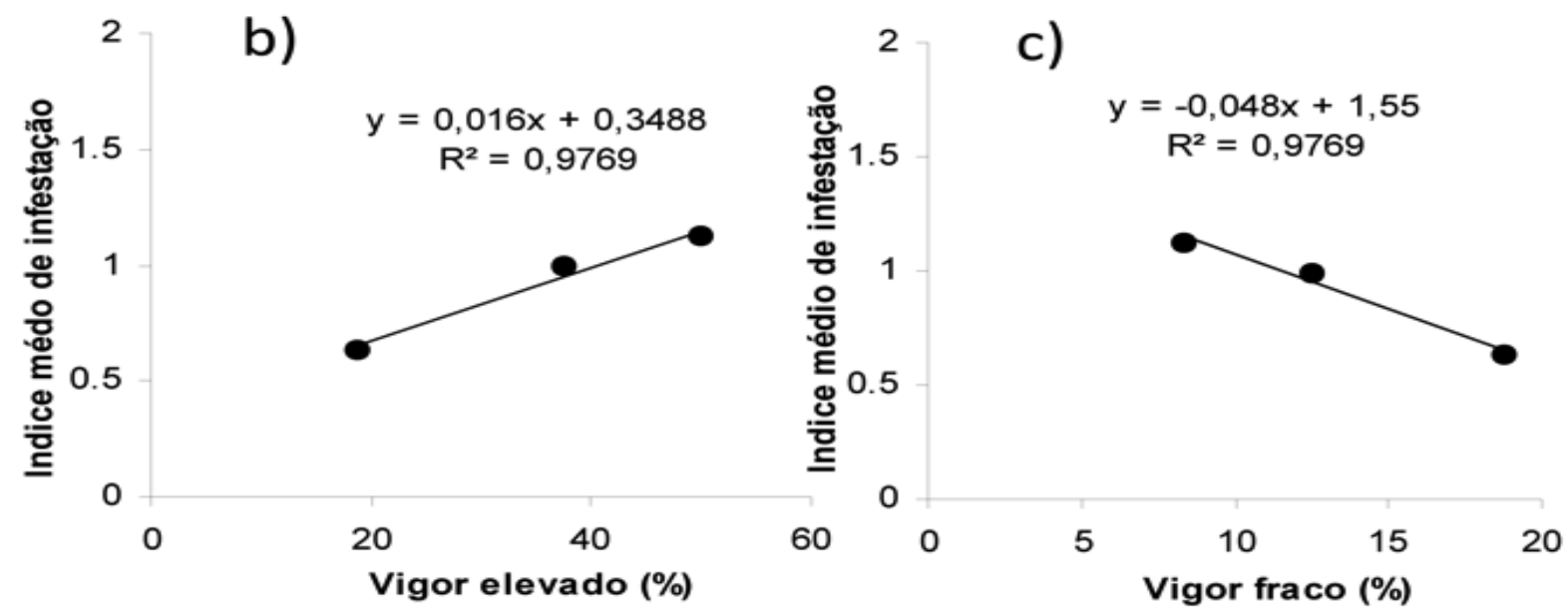


\section{Curva de voo dos machos}

Foi capturado um total de 263 machos de P. citri, durante o estudo, tendo a média das capturas variado entre 0 e 24,5 machos/armadilha/semana (Figura 6). Registaram-se períodos de oscilação muito rápida dos valores máximos, a partir de março, altura em que a temperatura média apresentou pequenas oscilações. Os picos de captura coincidiram com a mudança dos difusores de

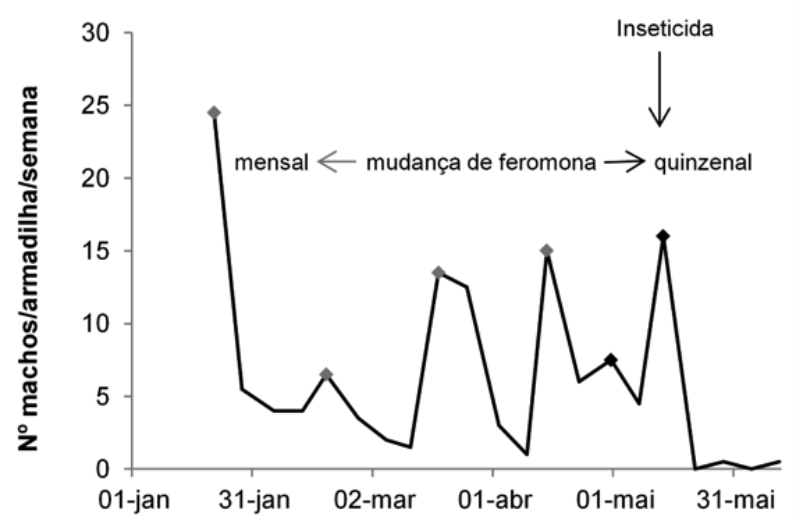

Figura 6 - Curva de voo dos machos de Planococcus citri (Risso) (Hemiptera, Pseudococcidae) com base na captura em armadilhas sexuais. feromona. As capturas médias reduziram-se acentuadamente para valores próximos de zero, após o tratamento com clorpirifos realizado a 16 de maio.

Comparação do desenvolvimento de Planococcus citri em duas variedades de cafeeiro, com diferente suscetibilidade à ferrugem-alaranjada

O número de indivíduos que se instalou em cada uma das fases de desenvolvimento do cafeeiro foi sempre inferior na variedade Catimor CIFC 45, comparativamente à variedade Caturra CIFC 19/1, exceto no caso dos machos (Figura 7). A percentagem de indivíduos nos vários instares manteve-se quase sempre próxima de $90-100 \%$, na variedade Caturra CIFC 19/1. No caso da variedade resistente à ferrugem-alaranjada (Catimor CIFC 45), o mesmo parâmetro registou marcada redução a partir do segundo instar, para valores inferiores a $50 \%$.

A temperatura média mensal variou entre $22^{\circ} \mathrm{C}$ e $25^{\circ} \mathrm{C}$, durante o período de observação. Os valores da temperatura média diária mais elevada $\left(29,5^{\circ} \mathrm{C}\right)$ e mais baixa $\left(19,5^{\circ} \mathrm{C}\right)$ foram registados durante $\mathrm{o}$ mês de maio (Figura 7). Nestas condições, P. citri necessitou de cerca de 42 dias para completar o desenvolvimento, em ambas as variedades de cafeeiro. Embora os ovos de P. citri tenham
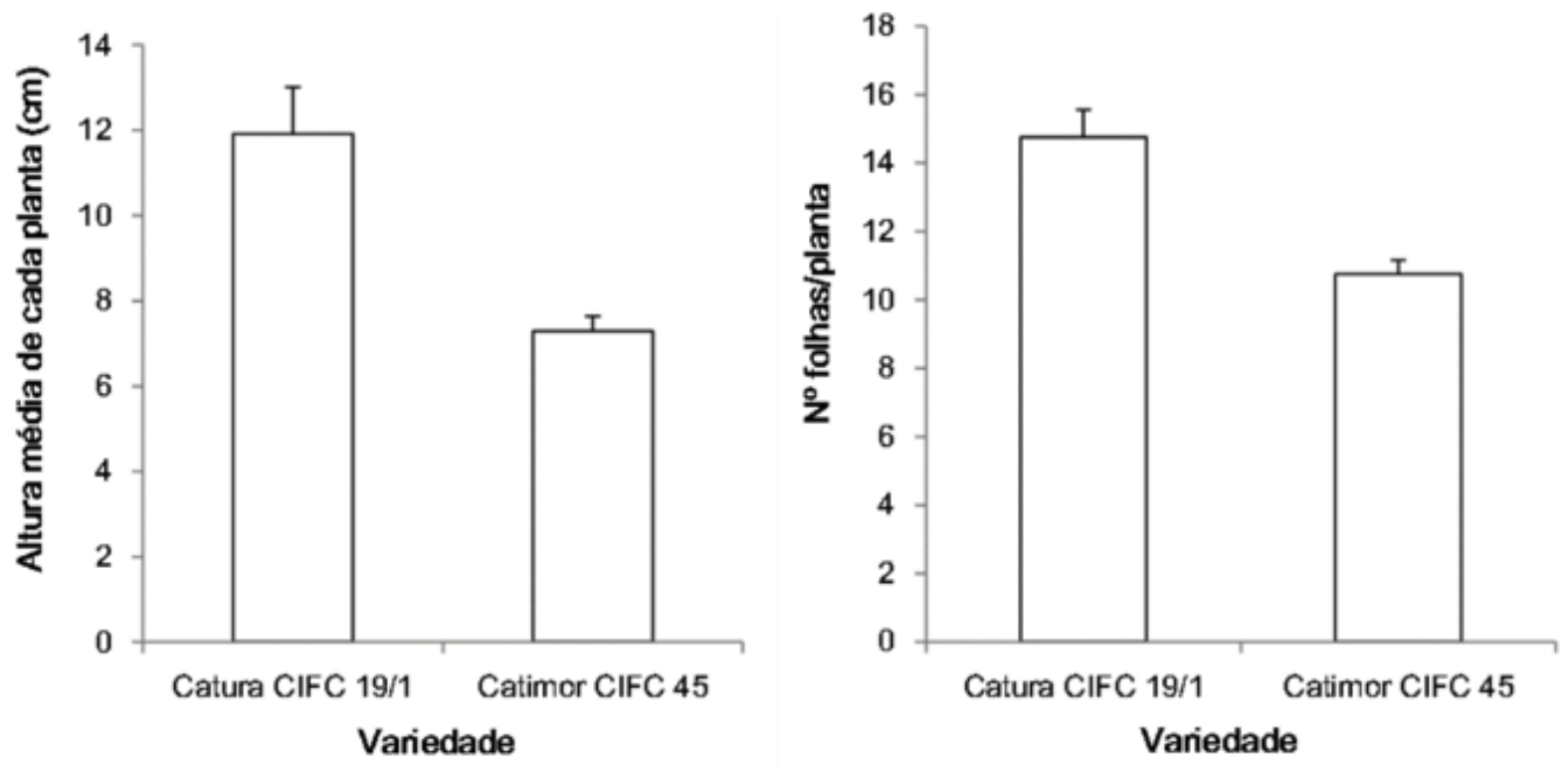

Figura 7 - Evolução da percentagem de indivíduos de Planococcus citri (Risso) (Hemiptera, Pseudococcidae), em cada um dos estados de desenvolvimento nas duas variedades de cafeeiro estudadas (A- Catimor CIFC 45; B- Caturra CIFC 19/1), e da temperatura na estufa (C) (CIFC, Oeiras). 

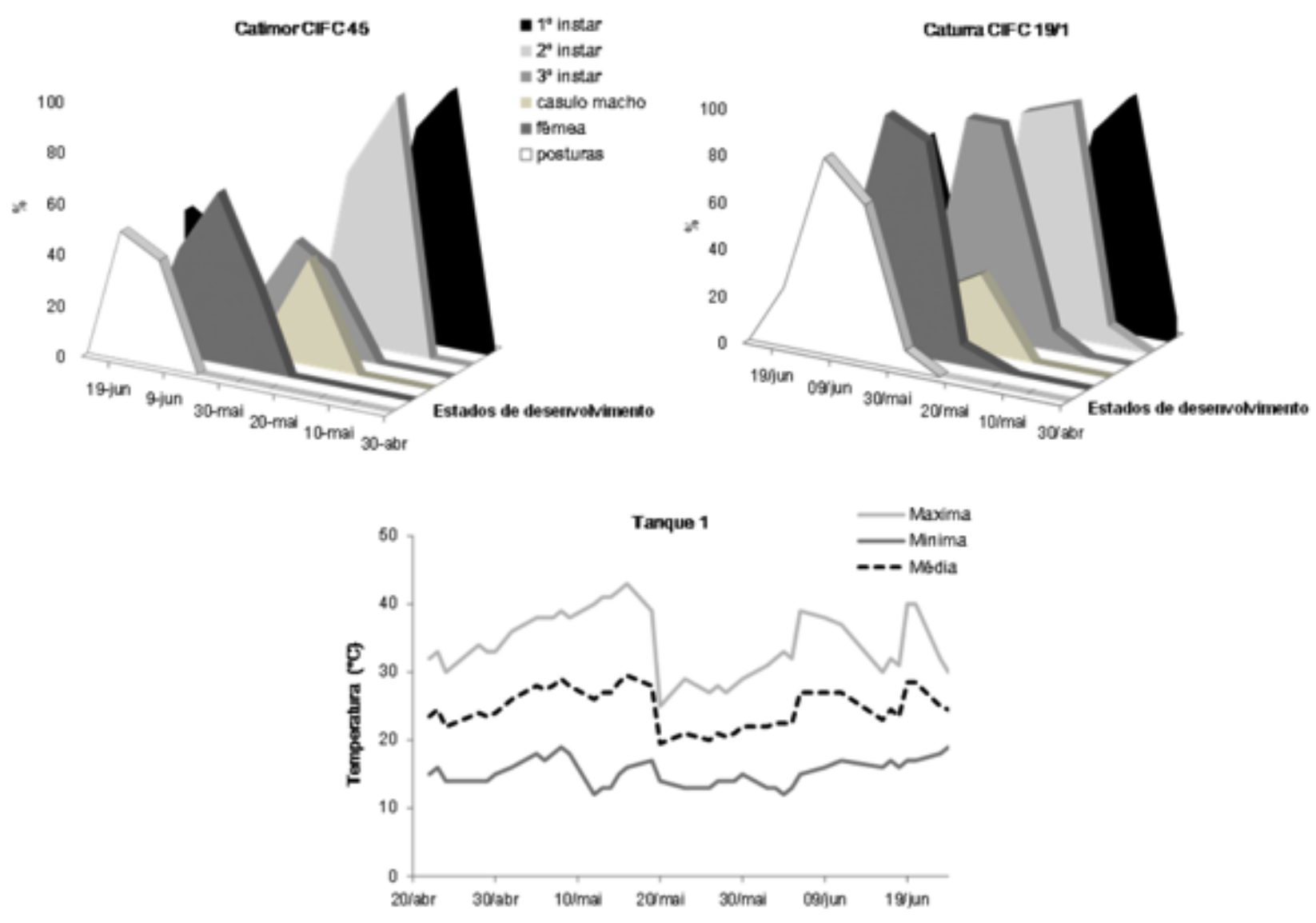

Figura 8 - Altura média de cada planta (A) e do número de folhas por planta (B) de cada uma das variedades de cafeeiro, no final do ensaio sobre o desenvolvimento de Planococcus citri (Risso) (Hemiptera, Pseudococcidae).

eclodido primeiro na variedade Caturra CIFC 19/1, não se verificaram diferenças significativas entre as duas variedades, quanto ao tempo necessário para completar uma geração.

$\mathrm{Na}$ variedade Catimor CIFC 45, 50\% das cochonilhas atingiram o estado adulto a 28 de maio. No caso de Caturra CIFC 19/1, esse mesmo valor foi atingido entre 22 e 28 de maio. Observaram-se diferenças significativas $\left(\mathrm{t}_{13,6}=-5,553, \mathrm{p}<0,001\right)$ no número médio de cochonilhas por planta entre as variedades Catimor CIFC $45(1,2 \pm 0,3)$ e Caturra CIFC 19/1 (5,8 $\pm 0,8)$, na semana em que se registou o pico de fêmeas adultas em cada variedade (4 junho e 28 maio, respetivamente).
O vigor vegetativo das duas variedades de cafeeiro no final do ensaio diferiu significativamente entre si (Figura 8), quer com base na altura de cada planta $(\mathrm{cm})\left(\mathrm{t}_{13}=4,015 ; \mathrm{p}=0,001\right)$, quer no número de folhas $\left(\mathrm{t}_{16}=-4,412 ; \mathrm{p}<0,001\right)$. As plantas da variedade Caturra CIFC 19/1 apresentaram sempre melhores condições para o desenvolvimento das cochonilhas (Figura 8), uma vez que cresceram mais rapidamente e apresentaram maior número de folhas do que a variedade Catimor CIFC 45 (Figura 9).

Não se detetou presença de cochonilhas nas raízes de qualquer variedade de cafeeiro, no final do ensaio. 

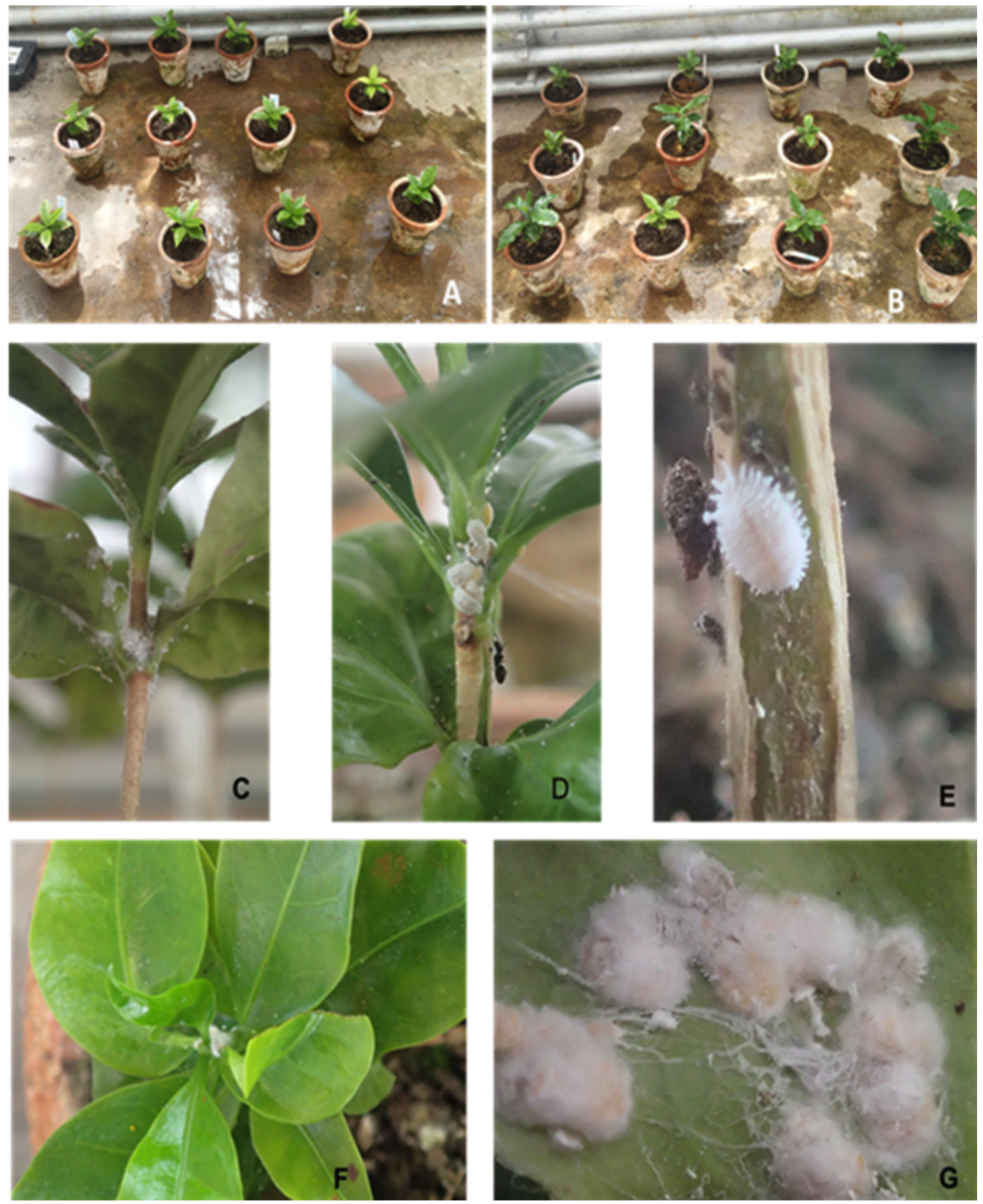

Figura 9 - Ensaio de desenvolvimento de Planococcus citri (Risso) (Hemiptera, Pseudococcidae) em cafeeiro (CIFC, Oeiras) em condições de estufa. A e B- desenvolvimento das plantas da variedade Catimor CIFC 45 e Caturra CIFC 19/1 respetivamente; C- exúvias e casulos de macho observado nas plantas da variedade Catimor CIFC 45; D- zona de instalação das cochonilhas; F- atrofiamento das folhas; E e G- fêmeas em posturas no tronco e na página inferior das folhas. 


\section{DISCUSSÃO}

Ambas as espécies de cochonilhas-algodão identificadas no presente estudo estão referenciadas em cafeeiro. Planococcus citri é referida por diferentes autores em vários países produtores (Le Pelley, 1968; Lavabre, 1970; Ferrão et al., 1971; Santa-Cecília et al., 2002) e P. madeirensis foi assinalada por Martínez et al. (2007) em Cuba.

Anagyrus pseudococci (Girault) está referenciado no Brasil como parasitóide de P. citri em cafeeiro (Costa et al., 2009). São poucas as referências a formigas em associação com cochonilhas-algodão em cafeeiro. Venkataramiah \& Rehman (1989) referem uma espécie não identificada de Crematogaster, na Índia. Das três espécies identificadas no presente estudo, a que foi observada em associação direta com cochonilhas-algodão também pertence ao mesmo género, C. auberti. Lasius grandis e T. simrothi têm sido referenciadas noutras plantas hospedeiras, associadas a cochonilhas-algodão (e.g., Pekas et al., 2011; Sharaf \& Aldawood, 2011).

Como medida do nível de infestação das plantas de cafeeiro por cochonilhas-algodão, o índice de infestação definido no presente trabalho traduziu com maior precisão a evolução da respetiva densidade populacional, em comparação com a percentagem de plantas infestadas, em particular no caso das densidades populacionais mais elevadas. De facto, contrariamente à relativa estabilidade observada na percentagem de plantas infestadas, em grande parte do período experimental, o índice de infestação evidenciou claro aumento ao longo do tempo, sobretudo a partir de meados de março, nas três variedades de cafeeiro (Figura 1). No entanto, verificou-se existir elevada relação entre os dois parâmetros, no caso das variedades Catimor e Sarchimor, embora nesta última sobretudo para valores do índice de infestação inferiores a 1,3 (Figura 2).

As diferenças observadas na dinâmica de infestação das cochonilhas-algodão, nas três variedades de cafeeiro estudadas, refletem, por um lado, os diferentes níveis de infestação inicial (Sarchimor > Catimor > Catuaí) e, por outro, possíveis diferenças de suscetibilidade entre variedades. O nível de infestação evoluiu, em termos relativos, de forma idêntica nas três variedades até meados de março, refletindo os diferentes níveis iniciais, tendo registado um aumento mais acentuado na variedade Catimor. O nível de infestação desta variedade ultrapassou, por isso, o da variedade Sarchimor, em meados de maio, imediatamente antes da realização do tratamento inseticida. $\mathrm{O}$ aumento do nível de infestação observado nas três variedades a partir de meados de março está possivelmente relacionado com o aumento da temperatura média diária, coincidindo também com o aumento do número de plantas em floração. Contudo, a maior taxa de crescimento verificada na variedade Catimor só pode ser explicada com base em características que diferenciam esta variedade das restantes e que a tornaram mais favorável ao crescimento da população de cochonilhas-algodão. O facto das diferenças na dinâmica de infestação só se terem evidenciado a partir de meados de março sugere que o fator responsável por essas diferenças seja de natureza fenológica, possivelmente associado à frequência de frutos em crescimento (Figura 3). Por outro lado, verificou-se que as diferenças registadas entre variedades de cafeeiro no índice médio de infestação estavam relacionadas com o vigor vegetativo, ou seja, o vigor vegetativo favoreceu o crescimento das populações de cochonilhas-algodão.

No caso de Catimor, o aumento do nível de infestação foi acompanhado por um aumento da percentagem de plantas infestadas (Figura 1), enquanto nas variedades Sarchimor e Catuaí esteve associado ao aumento do índice de infestação das plantas infestadas. Diferenças na estrutura das populações de cochonilhas-algodão, entre variedades, nomeadamente a possibilidade de maior proporção de fêmeas em postura e de primeiros instares, em meados de março, poderá ter conduzido a uma maior taxa de dispersão das cochonilhas e, consequentemente, aumento da percentagem de plantas infestadas. A este respeito, é de referir que a maior ou menor proximidade entre plantas vizinhas poderá facilitar mais ou menos essa dispersão, nomeadamente por permitir ou não a passagem de primeiros instares das plantas infestadas para as plantas vizinhas, através de ramos em contacto.

O número de insetos capturados em armadilhas sexuais depende de diversos fatores, nomeadamente o tipo de feromona e a respetiva taxa de difusão, o tipo de armadilhas e sua localização, a 
densidade populacional do inseto e as condições climáticas. Em condições de estufa, é de esperar que a taxa de difusão de feromona e a área de ação da armadilha sejam menores do que ao ar livre. No presente trabalho, a curva de voo dos machos de $P$. citri não apresentou um padrão bem definido, evidenciando vários picos que não correspondem apenas a variações da densidade populacional dos machos, refletindo também a influência das variações bruscas da temperatura máxima, da diminuição da taxa de difusão de feromona, ao longo do tempo, após cada mudança de difusor, e do facto de se estar a amostrar uma pequena população de cochonilhas num interior de uma estufa. Foi possível, no entanto, delimitar fases no ciclo biológico da cochonilha, nomeadamente relacionadas com períodos de reprodução. Por exemplo, o pico de capturas registado em meados de março coincidiu com o momento a partir do qual se verificou o incremento do índice de infestação nas três variedades estudadas de cafeeiro, sugerindo que este pico está relacionado com um período de reprodução que conduziu a aumentos da densidade populacional de P. citri. Por outro lado, a monitorização dos machos através das armadilhas sexuais mostrou, também, ser sensível à redução populacional registada após o tratamento inseticida.

A oportunidade do tratamento inseticida com clorpirifos foi determinada com base no aparecimento do maior número de formas sensíveis. O tratamento foi aparentemente eficaz, uma vez que o índice de infestação baixou para valores próximos de zero, no caso das variedades Sarchimor e Catuaí. Na variedade Catimor, o impacto do tratamento foi menor, possivelmente devido ao maior índice de infestação desta variedade, antes do tratamento, tendo permitido a sobrevivência de algumas fêmeas em postura, protegidas no interior das colónias. Não obstante a aparente eficácia do tratamento realizado, seria recomendável estudar a possibilidade de desenvolver uma estratégia de proteção alternativa, baseada na luta biológica, por exemplo, com largadas aumentativas do encirtídeo $A$. sp. próx. pseudococci e/ou do coccinelídeo Cryptolaemus montrouzieri Mulsant, a depender da intensidade de ataque e da estrutura da população de cochonilha-algodão. Anagyrus sp. próx. pseudococci é parasitóide de P. citri e a sua presença foi detetada nas estufas do CIFC, durante o presente estudo. Esta espécie e $C$. montrouzieri estão disponíveis comercialmente em Portugal. Para além dos efeitos secundários, em termos de toxidade para o Homem e ambiente, em particular para os organismos aquáticos, a utilização do clorpirifos, no combate às cochonilhas-algodão nas estufas do CIFC, apresenta o inconveniente de poder interferir nos respetivos objetivos de investigação, nomeadamente através da presença de resíduos nas folhas que são utilizadas para inocular a ferrugem-alaranjada.

No ensaio comparativo sobre o desenvolvimento de $P$. citri nas duas variedades de cafeeiro com diferentes níveis de resistência à ferrugem-alaranjada, as diferenças observadas registaram-se mais ao nível do número de indivíduos instalados por planta e da taxa de sobrevivência do que na duração do desenvolvimento. Não foi possível contabilizar, com precisão, a duração de cada estado de desenvolvimento, uma vez que as observações foram semanais. No entanto, estima-se que a duração média do desenvolvimento foi de 42 dias, para ambas as variedades. Correa et al. (2008) referem que, à temperatura ótima de $25^{\circ} \mathrm{C}$, a duração do desenvolvimento de $P$. citri em cafeeiro Arábica (cv. Mundo Novo) foi, em média, de 8,5 dias para o primeiro instar, 7,1 dias para o segundo instar e 8,1 dias para o terceiro instar. A longevidade média das fêmeas adultas foi de 59,6 dias.

A diminuição do número de indivíduos, a partir do primeiro e segundo instares, observada na variedade Catimor CIFC 45, sugere elevada mortalidade dos primeiros instares e/ou maior proporção de machos. De facto, esta variedade apresentou número mais elevado de machos/casulos de cochonilha-algodão do que a variedade Caturra CIFC 19/1.

O desenvolvimento médio das plantas em termos de altura e número de folhas foi sempre superior na variedade Caturra CIFC 19/1, comparativamente à variedade Catimor CIFC 45 (Figuras $8 \mathrm{e}$ 9). As diferenças observadas entre variedades, no número de cochonilhas por planta, parecem estar relacionadas com o vigor vegetativo das plantas. No entanto, não é possível concluir se as diferenças de vigor entre as duas variedades refletem diferenças genéticas, ou resultam apenas de condicionalismos associados ao processo de obtenção das plantas. 
A localização das cochonilhas nas plantas de cafeeiro variou em função do sexo e do estado de desenvolvimento. As fêmeas em postura foram colocadas nas folhas mais jovens, mas os primeiros instares instalaram-se junto ao pedúnculo das folhas (Figura 9). As exúvias e os casulos dos machos encontravam-se sempre na zona de inserção das folhas (Figura 9) ou na página inferior das folhas cotiledonares, que são mais arredondadas e conferem maior enrolamento e com isso menos luz, ou ainda na parte de fora do rebordo do vaso. As fêmeas, no momento de postura, deslocaram-se para as folhas junto ao solo e instalaram-se na página inferior ou ao longo do caule, depois das primeiras folhas (Figura 9). As observações efetuadas permitiram verificar que o pedúnculo das folhas e as folhas jovens, ainda coladas umas às outras, eram os locais com maior probabilidade de encontrar cochonilhas, nomeadamente quando o nível populacional era muito baixo, sugerindo que nestes locais a qualidade e a quantidade de seiva é máxima.

\section{CONCLUSÕES}

Foram identificadas duas espécies de cochonilhas-algodão associadas a cafeeiro arábica, nasestufas do CIFC, nomeadamente P. citri e P. madeirensis, sendo a primeira, aparentemente, a espécie dominante.

Os resultados sugerem que a variedade Catimor é mais suscetível do que a Sarchimor, uma vez que, apesar de ter registado nível inicial de infestação inferior ao desta variedade, atingiu níveis superiores no final do período experimental. Os resultados obtidos indicam, igualmente, que o nível de infestação está diretamente relacionado com o vigor vegetativo das plantas.

A monitorização dos machos de $P$. citri, através de armadilhas sexuais, pode ser utilizada como ferramenta para auxiliar na deteção dos períodos críticos do ciclo biológico da cochonilha, nomeadamente em termos de reprodução e na avaliação da eficácia dos métodos de proteção.

Não se observaram diferenças na duração do desenvolvimento de P. citri, entre as variedades de cafeeiro Catimor CIFC 45 (resistente à ferrugem-alaranjada) e Caturra CIFC 19/1 (suscetível). No entanto, a taxa de colonização e o número de cochonilhas que completaram o desenvolvimento foi superior na variedade Caturra CIFC 19/1. As diferenças observadas entre as duas variedades, relativamente a estes parâmetros, estão aparentemente relacionadas com diferenças de vigor vegetativo.

\section{AGRADECIMENTOS}

Aos Professores Agatino Russo e Gaetana Mazzeo (Universidade de Catânia, Itália), pela confirmação da identificação das espécies de cochonilhas; à Mestre Vera Zina (ISA, Universidade de Lisboa), pela identificação das espécies de formigas; à Doutora Maria do Céu Silva e ao Eng. Vítor Várzea (CIFC, Oeiras), por terem apoiado a realização deste estudo. Este trabalho foi realizado no âmbito do projeto UID/AGR/00239/2013, financiado pela FCT.

\section{REFERÊNCIAS BIBLIOGRÁFICAS}

Barrera, J.F. (2008) - Coffee pests and their management. In: Capinera, J.L. (Ed.) - Encyclopedia of entomology. Netherlands, Springer, pp. 961-998. [cit. 2014-01]. http://www2.tapecosur.edu.mx/mip/Publicaciones/pdf/ Coffeepetsandtheirmanagement.pdf

Ben-Dov, Y. (2014) - ScaleNet, scales on a host. [cit. 2014-08-26]. http://www.sel.barc.usda.gov/scalecgi/scaleson. exe?family=rubiaceae\&scalefamily=Pseudococcidae\&genus $=$ coffea\&scalegenus $=\& s p e c i e s=$

Collingwood, C. \& Prince, A. (1998) - A guide to ants of continental Portugal (Hymenoptera: Formicidae). Boletim da Sociedade Portuguesa de Entomologia, supl. n.. 5, p. 1-49. 
Correa, L.R.B.; Santa-Cecília, L.V.C.; Souza, B. \& Cividanes, F.J. (2008) - Efeito de Diferentes temperaturas e exigências térmicas da cochonilha-branca Planococcus citri (Risso, 1813) (Hemiptera: Pseudococcidae) em cafeeiro. Arquivos do Instituto Biológico, vol. 75, n. 1, p. 53-58.

Costa, J.N.M.; Teixeira, C.A.D.; Sallet, L.A.P. \& Gama, F.C. (2009) - Cochonilhas ocorrentes em cafezais de Rondônia. Embrapa Rondônia, Circular Técnica, n.o 110, p 1-6.

Ferrão, A.P.S.F.; Carvalho, J.P. \& Rodrigues, A.H. (1971) - Manual das pragas do cafeeiro. Nova Lisboa, Instituto de Investigação Agronómica de Angola, 246 p.

Fornazier, M.J.; Perini, J.L.; De Muner, L.H.; Machado, V.L.; Mazzo, G.; Santa-Cecília, L.V.C.; Souza, J.C. \& Daun, S.C. (2000) - Cochonilha branca da roseta em café conilon (Coffea canephora) no Espírito Santo. Congresso Brasileiro de Pesquisas Cafeeiras, Rio de Janeiro, Brasil, p. 176-177.

ICO [International Coffee Organization] (2016) - Trade statistics Tables: Total production by exporting countries [cit. 2017-01-11]. http://www.ico.org/prices/po-production.pdf

Kumar, P.K.V.; Vasudev, V.; Seetharama, H-G.; Irulandi, S. \& Sreedharan, K. (2008) - Attendant ants and activity of Spalgis epius. Journal of Coffee Research, vol. 36, n. 1-2, p. 38-45.

Lavabre, E.M. (1970) - Insectes nuisibles des cultures tropicales (cocoayer, cafeier, calatier, poivrier, theier). Tecniques agricoles et productions tropical. Paris, Ed. Maisonneuve \& Lorose, $276 \mathrm{p}$.

Le Pelley, R.H. (1968) - Las plagas del café. Agricultura tropical. Barcelona, Ed. Labor s.a., 693 p.

Martínez, M.A.; Surís, M. \& Blanco, E. (2007) - Fauna de chinches harinosa (Hemiptera: Pseudococcidae) asociada a plantas de interes: III. Cafeto y cacao. Revista de Protección Vegetal, vol. 22, n. 2, p. 85-88.

Pekas, A.; Tena, A.; Aguilar, A. \& Garcia-Marí, F. (2011) - Spatio-temporal patterns and interactions with honeydew-producing Hemiptera of ants in a Mediterranean citrus orchard. Agricultural and Forest Entomology, vol. 13, n. 1, p. 89-97. http://dx.doi.org/10.1111/j.1461-9563.2010.00501.x

Pereira, A.P. \& Guimarães, L. (2010) - O CIFC e a importância do Hibrido de Timor na cafeicultura mundial. In: Martins, A.C. \& Albino, T. (Eds.) Viagens e missões científicas nos trópicos 1883-2010. Lisboa, Instituto de Investigação Cientifica Tropical, p. 119-121.

Rahiman, P.A.; Naik, P.R. \& Vijayalakshmi, C.K. (2010) - Field evaluation of coffee mealy bug parasitoid, Leptomastix dactylopii (How.) in Wayanad district of Kerala, India. Gregor Mendel Foundation Journal, vol. 1, p. 62-67.

Santa-Cecília, L.V.C.; Reis, P.R. \& Souza, J.C. (2002) - Sobre a nomenclatura das espécies de cochonilhas-farinhentas do cafeeiro nos Estados de Minas Gerais e Espírito Santo. Neotropical Entomology, vol. 31, n. 2, p. 333-334. http://dx.doi.org/10.1590/S1519-566X2002000200024

Santa-Cecília, L.V.C.; Souza, B.; Prado, E.; Souza, J.C. \& Fornazier, M.J. (2005) - Cochonilhas-farinhentas em cafeeiros: reconhecimento e controle. Circular Técnica, n.․189, p. 1-4.

Sharaf, M.R. \& Aldawood, A.S. (2011) - First occurrence of Solenopsis Westwood 1840 (Hymenoptera: Formicidae), in the Kingdom of Saudi Arabia, with description of a new species S. saudiensis sp. n. Annals de la Société Entomologique de France, vol. 47, p. 474-479.

Thuy, N.T.; Vuong, P.T. \& Hung, H.Q. (2011) - Composition of scale insects on coffee in Daklak, Vietnam and reproductive biology of Japanese mealybug, Planococcus kraunhiae Kuwana (Hemiptera: Pseudococcidae). ISSAAS Journal, vol. 17, n. 2, p. 29-37.

Triapitsyn, S.V.; González, D.; Danel, B.; Vickerman, D.B.; Noyes, J.S. \& Ernest, B.W. (2007) - Morphological, biological, and molecular comparisons among the different geographical populations of Anagyrus pseudococci (Hymenoptera: Encyrtidae), parasitoids of Planococcus spp. (Hemiptera: Pseudococcidae), with notes on Anagyrus dactylopii. Biological Control, vol. 41, n. 1, p. 14-24. http://dx.doi.org/10.1016/j.biocontrol.2006.12.013

Venkataramaiah, G.H. \& Rehman, P.A. (1989) - Ants associated with the mealybugs of coffee. Indian Coffee, vol. 43, n. 90, p. 13-14.

Williams, D.J. \& Granara de Willink, M.C. (1992) - Mealybugs of Central and South America. London, England, CAB International, $635 \mathrm{pp}$.

Williams, D.J. \& Watson, G.W. (1988) - The scale insects of the tropical South Pacific Region. Part 2-The mealybugs (Pseudococcidae). Wallingford, CAB International, $267 \mathrm{p}$. 